\title{
HaP/SBA-3 Nanostructured Composite to Remove Fluoride Effectively from Contaminated Water
}

ISSN: 2576-8840

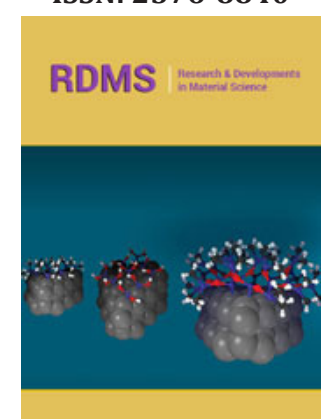

*Corresponding author: Oscar A Anunziata, Center for Research in Nanoscience and Nanotechnology (NANOTEC), Córdoba Regional Faculty, National Technological University, Córdoba, Argentina

Submission: 些 November 11, 2021

Published: 潐 November 29, 2021

Volume 16 - Issue 2

How to cite this article: Jorgelina Cussa Claudia G Lopez, Oscar A Anunziata. HaP/SBA-3 Nanostructured Composite to Remove Fluoride Effectively from Contaminated Water. Res Dev Material Sci. 16(2). RDMS.000883. 2021.

DOI: $10.31031 /$ RDMS.2021.16.000883

Copyright@ Oscar A Anunziata. This article is distributed under the terms of the Creative Commons Attribution 4.0 International License, which permits unrestricted use and redistribution provided that the original author and source are credited.

\section{Jorgelina Cussa, Claudia G Lopez and Oscar A Anunziata*}

Center for Research in Nanoscience and Nanotechnology (NANOTEC), Córdoba Regional Faculty, National Technological University, Córdoba, Argentina

\begin{abstract}
Highly ordered pore mesoporous silica composites, like SBA-3 and hydroxyapatite (HaP) nanocrystals, characterized by X-ray diffraction (XRD), Fourier Transform Infrared Spectroscopy (FTIR), Scanning Electron Microscopy (SEM), Transmission Electron Microscopy (TEM) and textural properties, were successfully applied to remove fluoride from contaminated water. The proposed procedure to prepare HaP/SBA-3 was successful, which acts as supports to anchor the HaP crystals, in nanometer-scale $(<2 \mathrm{~nm})$, with higher fluoride retention from contaminated water. The free $\mathrm{OH}$ - groups of $\mathrm{HaP}$ nanocrystals, within the host, facilitated the high-performance fluoride trapping. The fluoride retention activity was much higher than that of pure HaP and the composites HaP/SBA-15 and HaP/MCM-41.
\end{abstract}

Keywords: F- retention; Contaminated water; HaP/SBA-3; Nanocomposites

\section{Introduction}

Nanostructured materials, based on silica (MCM, SBA [1,2]) and ordered mesoporous carbon (OMC), such as CMK-1, CMK-3 [3] and Multi-Walled Carbon Nanotubes (MWCN) [4], because of their large surface area, pore volume and possibility to vary the pore size, offer a great opportunity for their application in various fields, from industrial processes to biomedical engineering, air and water pollution decontamination and energy reservoirs. Recently, we have developed a techniqueof preparation of monocrystalline hydroxyapatite (HaP) , and by the same procedure, but in the presence of the respective hosts, forming in situ composites such as HaP/MCM-41 and HaP/SBA- 15 composites [5]. Numerous approaches for fluoride removal from contaminated water, such as precipitation-coagulation [6], membrane technology [7,8], ion exchange [9] and adsorption [10,11]. Among the above methods, fluoride adsorption on adsorbents is attracting increasing attention due to its advantages of convenient design and operation, and low cost [12]. Different types of adsorbents, such as metal adsorbents [13], carbon adsorbents [14], carbon materials [15], natural materials [16], and biosorbents [17], have been used to remove fluoride from water. MOFs have also been used as new adsorbents for fluoride adsorption due to their surface functional groups and ordered atomic arrangement [18]. Calcium phosphate apatites are compounds of the formula $\mathrm{Ca} 5$ (P04)3X, where $\mathrm{X}$ can be an $\mathrm{F}^{-}$ion (fluorapatite, $\mathrm{FaP}$ ), $\mathrm{OH}^{-}$(hydroxyapatite, $\mathrm{HaP}$ ), or a $\mathrm{Cl}$ - ion (chlorapatite). The ion is substituted by another ion of the same sign but of different charge. Neutrality is maintained by substitutions of ions with different charges or vacancies [19]. Studied the crystallization of HaP (hydroxyapatite) on polymers containing - C-N groups, from supersaturated solutions of HaP has been reported [20]. The growth of hydroxyapatite on silica gelsin the presence of organic additives was studied by Rivera-Muñoz et al. [21]. Laghzizil et al. [22] have correlated the results of HaP fluorination with the physicochemical properties of HaP. Recently, morphological modifications of hydroxyapatite using fatty acids as an organic modifier have been reported [23]. The goal of this work is the successful 
development of a HaP/SBA-3 nanostructured composite material, by exploiting the small pore size of SBA-3 ( $3 \mathrm{~nm})$, with very large surface are, to embed nanonized $\mathrm{HaP}$ inside the pores, with size less than 2 nanometers, and apply the Hap/SBA-3 nanocomposite in the decontamination of fluorides from the polluted water.

\section{Experimental}

\section{Materials synthesis}

The procedure designed for the synthesis of SBA-3 was as follows [24,25]: the surfactant (cetyltrimethylammonium bromide), was added to water and $\mathrm{HCl}$; afterwards, $3 \mathrm{~g}$ of TEOS was also added, stirring to obtain a mixture with a molar composition of: TEOS:H2O:HCl:CTAB=1:150:9:0.15. After 60min, the white precipitate was filtered, washed and dried, and then the surfactant was extracted using ethanol at reflux for $4 \mathrm{~h}$, and subsequentlycalcined at $550{ }^{\circ} \mathrm{C}$ in air for $2 \mathrm{~h}$. Nanonized HaP was obtained using solutions of varying concentration $1-0.6 \mathrm{M} \mathrm{CaCl} 2$ in $2 \mathrm{H} 2 \mathrm{O}$ (a) and 1.8-2.30 M K2HPO4 (b) and then Benzalkonium Chloride 10\% Solution (Alkylbenzyldimethylammonium chloride, C6H5CH2N(CH3)2RCl, R=C18H37), in double distilled water was added to prevent the growth of HaP crystals (ex-situ). Theresulting solution $(\mathrm{pH}=9)$ was stirred vigorously for $2 \mathrm{~h}$ at $65{ }^{\circ} \mathrm{C}$, obtaining nanometric HaP (4-5nm). For synthesizing HaP-Host, the same procedure was performed, with the modification that was added to the mixture of solutions (a) and (b), SBA-3 (50-70\% of HaP wt/ wt with respect to thehost), after the first hour of reaction which was used to prepare HaP ex situ. The suspensions of HaP/SBA-3 were stirred vigorously for $4 \mathrm{~h}$, at $60^{\circ} \mathrm{C}$, filtered, washed with triple distilled carbon dioxide-free water, and then dried at $100{ }^{\circ} \mathrm{C}$ for $4 \mathrm{~h}$. Subsequently, the composite was activated by heating at 500 ${ }^{\circ} \mathrm{C}$ in $\mathrm{N} 2$ flow for $5 \mathrm{~h}$, and then calcined at $550{ }^{\circ} \mathrm{C}$ at a heating rate of $5{ }^{\circ} \mathrm{C} / \mathrm{min}$ from $100{ }^{\circ} \mathrm{C}$ for $4 \mathrm{~h}$. Hap/MCM-41 and HaP-SBA-15 [4], were also used in this study to compare the effectiveness of fluoride retention.

\section{Fluoride retention essay}

Experimental conditions: $2 \mathrm{~g}$ of $\mathrm{HaP}$ in $100 \mathrm{~mL}$ of $\mathrm{NaF}$ solution with an initial concentration of $9 \times 10^{-2} \mathrm{M}$ were used. The weight of the composite used was normalized to HaP. Fluoride ion solutions were prepared using a Teflon device with magnetic stirring, specially designed to bubble $\mathrm{N} 2$ to avoid $\mathrm{CO} 2$ contamination at $25{ }^{\circ} \mathrm{C}$. The $\mathrm{pH}$ of the solutions was measured with a Mettler $\mathrm{pH}$ meter; the instrument was calibrated with buffers of $\mathrm{pH}=4$ and 7.5. The concentration of $\mathrm{F}^{-}$ions was determined using an $\mathrm{F}^{-}$specific electrode, with a dynamic range between 1-300 ppm. In addition, trace amounts of $\mathrm{F}^{-}$were followed by FTIR. Fluoride retention capacity was evaluated in successive cycles at different times.

\section{Characterization}

Samples were characterized by means of powder X-ray diffraction in a X'Pert Pro PANalytical diffractometer equipped with a CuKa radiation source ( $\mathrm{k}=0.15418 \mathrm{~nm})$. N2 adsorption/ desorption isotherms were measured on ASAP 2020 equipment after degassing samples at $673 \mathrm{~K}$, determining textural properties such as surface area and pore volume; pore size distribution was estimated using Barrett, Joyner and Halenda (BJH) algorithm. TEM was recorded in a JEOL $2100 \mathrm{~F}$ microscope operated with an accelerating voltage of $200 \mathrm{kV}$ (point resolution of $0.19 \mathrm{~nm}$ ). FTIR studies were performed in a JASCO 5300 Fourier transform infrared spectrometer (FTIR).

\section{Results and Discussion}

\section{XRD, BET, SEM and HRTEM}

The surface area of the nanonized HaP measured by BET (N2) method was $96 \mathrm{~m}^{2} / \mathrm{g}$. The surface area of SBA-3 was $1064 \mathrm{~m}^{2} / \mathrm{g}$, and $750 \mathrm{~m}^{2} / \mathrm{g}$ for Hap/SBA-3 respectively. The pore diameter of the host was: $3.1 \mathrm{~nm}$ for SBA-3 and $\mathrm{a}_{0}$ of 3.56 (lattice parameter $\left(\mathrm{a}_{0}=2 \mathrm{~d}[100] / \mathrm{p} 3\right)$.). The compositeHaP/SBA-3 isotherms show a residual pore volume of $0.75-0.73 \mathrm{~mL}$ per gram. We can distinguish the presence of three signals in the symmetry of the hexagonal network by XRD, higher signal at hkl [100] $3.31 \mathrm{~nm}$, and lower [110] and [200] typical of the SBA-3 structure, indicating a highly ordered pore system with a high porosity (Figure 1a).
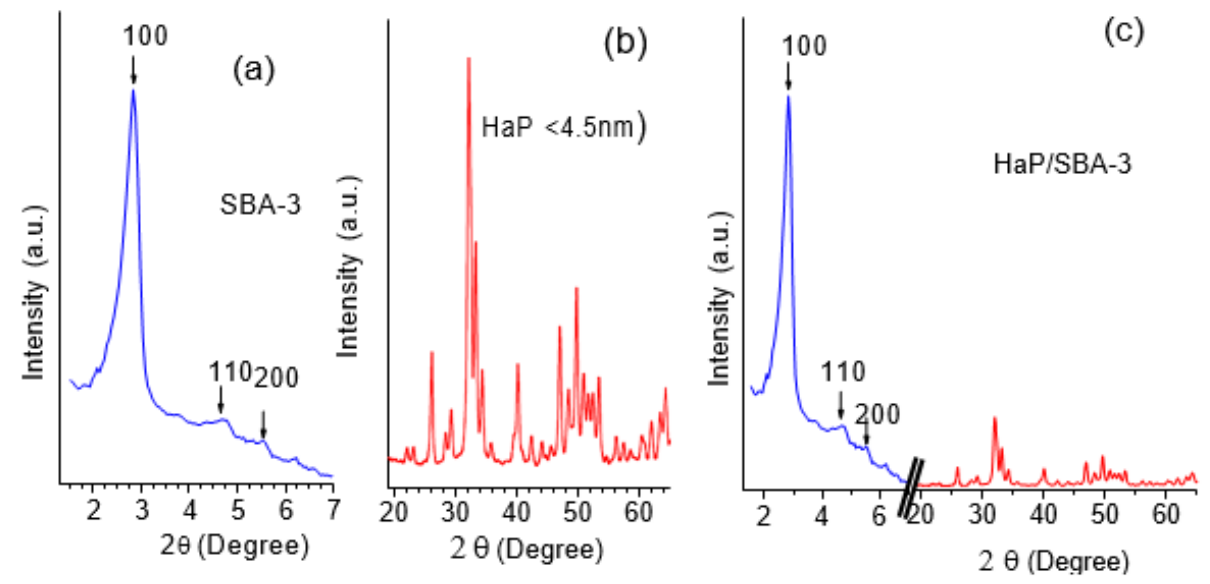

Figure 1: (a) SBA-3, (b) HaP, (c) HaP/SBA-3. 
The XRD pattern of the nanonized HaP prepared ex-situ and the HaP/SBA-3 composite areillustrated in Figure 1b \& 1c. Composite pattern diffraction peaks confirm a high crystallinity or long-range order structure in the nanostructured host SBA-3.

SEM studies (Figure 2a) show the size and shape of the HaP/ SBA-3 composite, which indicate a goodmorphology of the crystals,

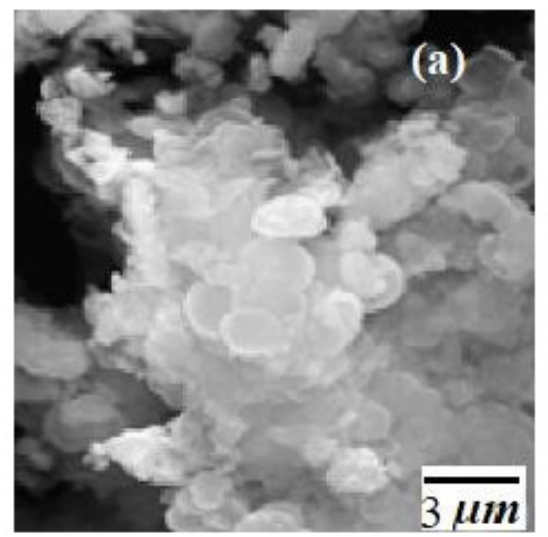

Figure 2: (a) SEM, (b) TEM of HaP/SBA-3.

\section{FTIR studies}

The FTIR analysis of HaP/SBA-3 (Figure 3) reveals bands assigned to SBA-3 and $\mathrm{HaP}$ in the composite.

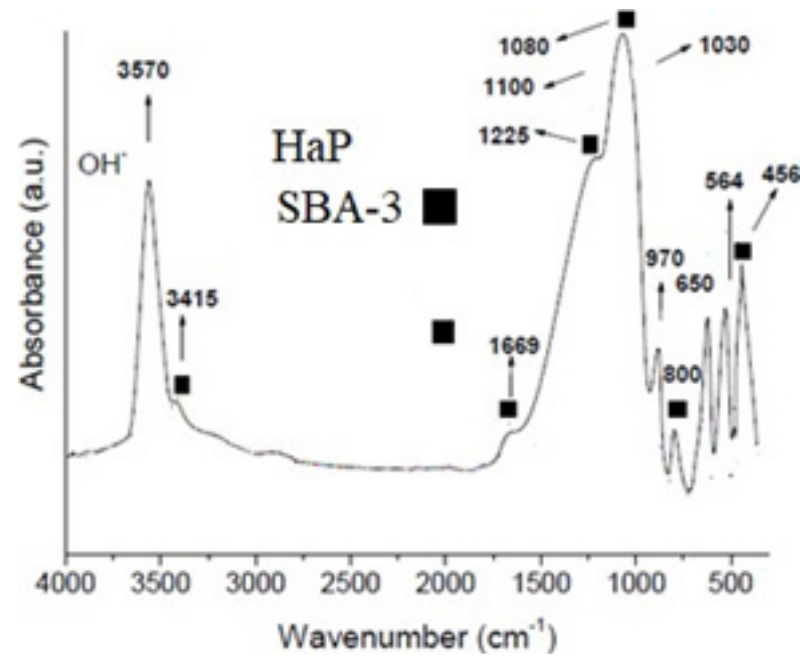

Figure 3: FTIR of HaP/SBA-3 nanocomposite.

The peak at $3570 \mathrm{~cm}^{-1}$ is associated with the $\mathrm{OH}$ stretching mode of $\mathrm{HaP}$ and at 3415 with $\mathrm{Si}-\mathrm{OH}$ streching. The signals at 1080, 1227 and $1669 \mathrm{~cm}^{-1}$ (asymmetric T-0, internal and external stretching respectively) are due to SBA-3. P04 appeared as a very strong band at $\sim 1030 \mathrm{~cm}^{-1}$. P-O stretching is found at $\sim 970 \mathrm{~cm}^{-1}$. The clearly defined bands at 650 and $564 \mathrm{~cm}^{-1}$ are ascribed to the O-P-O asymmetric deformation components. The band at $800 \mathrm{~cm}^{-1}$ is caused by SI-O symmetric stretching of SBA-3. The typical band followed for $\mathrm{F}^{-}$retention is that of $\mathrm{OH}^{-}$at $3570 \mathrm{~cm}^{-1}$ from $\mathrm{HaP}$ in the composite. with an absence of other phases caused by HaP. The shape of the HaP/SBA-3 crystals is practically spherulitic with a mean crystal size in the range of 1.5 to $2 \mu \mathrm{m}$. The HRTEM image illustrated in Figure $2 \mathrm{~b}$, detail the long-range hexagonal arrangement of HaP/SBA-3. Higher level reflections remain clearly detectable. Consequently, the nanostructured HaP crystals is within the nanostructure of the host.

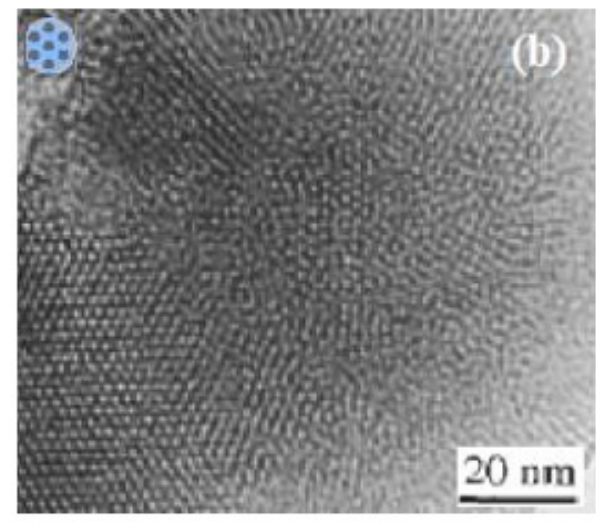

\section{Flouride retention}

To study the fluoride retention capacity of HaP/SBA-3 nanocomposite and its comparison with $\mathrm{HaP}, \mathrm{HaP} / \mathrm{MCM}-41$ and HaP/SBA-15 two strategies were employed. a: All fresh materials were evaluated in the function of contact time with a solution containing fluorides and the decrease of fluorides from $20 \%, 40 \%$, $60 \%$, $80 \%$, and $100 \%$. Thus, Table 1 shows a high $\mathrm{F}^{-}$retention capacity at short contact times with respect to the rest of the materials used, even with the mesoporous silica nanocomposites. The prepared nanosized HaP did not retain $100 \% \mathrm{~F}^{-}$even after several hours in contact with the solution.

Table 1: Fluoride retention (\%) at different contact time of various reservoirs.

\begin{tabular}{|c|c|c|c|c|}
\hline \multicolumn{5}{|c|}{ Contact Time, $\mathbf{h}$} \\
\hline F- Retention \% & HaP/SBA-3 & HaP/MCM-41 & HaP/SBA-15 & HaP* \\
\hline 20 & 0.5 & 8 & 5 & 7 \\
\hline 40 & 1 & 10 & 9 & 11 \\
\hline 60 & 2 & 13 & 10 & 16 \\
\hline 80 & 2.7 & 17 & 13 & 22 \\
\hline 100 & 4 & 20 & 16 & --- \\
\hline
\end{tabular}

*Prepared ex-situ

\section{Reusability}

HaP/SBA-3 was taken and its reusability was studied, in different cycles with a duration of $2 \mathrm{~h}$ and the percentage of retained $\mathrm{F}^{-}$and the amount free $\mathrm{OH}^{-}$bands of $\mathrm{HaP}$, in \% Absorbance Units, in the composite are reported. In Figure 4 it can be observed that after 5 cycles of $4 \mathrm{~h}$ duration thenanocomposite still retains $100 \%$ of $\mathrm{F}^{-}$ from fresh solutions. Inter-cycle, the nanocomposite was filtered, washed only with bi-distilled water and dried at $100{ }^{\circ} \mathrm{C}$, without 
any reactivation. The results suggest that in the fresh HaP/SBA-3 composite, the $\mathrm{OH}^{-}$of the HaP has remained intact because of high dispersion with the smaller size of the nanocrystals (less than $2 \mathrm{~nm}$ ) of $\mathrm{HaP}$ and thehigher proportion of free $\mathrm{OH}^{-}$per gram of the active material. The results also indicated that $\mathrm{HaP} / \mathrm{SBA}-3$ nanocomposite developed is not only active in terms of the time in contact with the solution to achieve $100 \% \mathrm{~F}^{-}$retention, but also that it remains active after several cycles, making ita highly attractive material for $\mathrm{F}^{-}$retention of contaminated water.

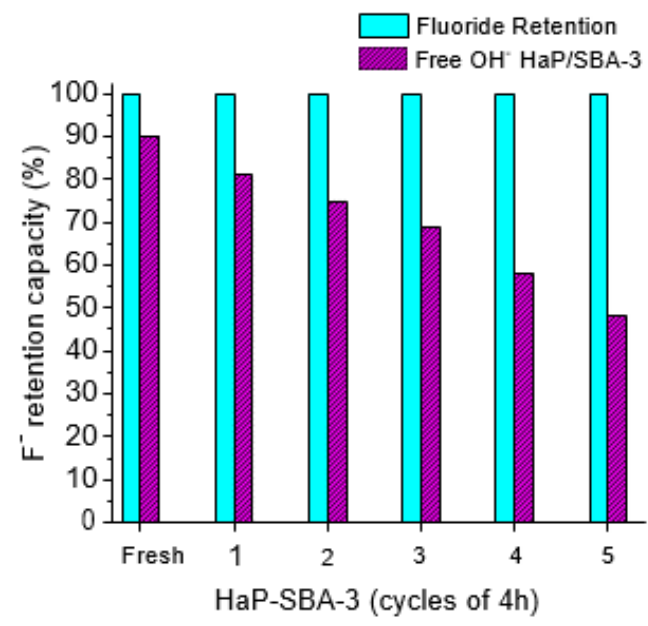

Figure 4: Capacity of $\mathrm{F}$ - retention and $\mathrm{OH}$ - free (\%) of Hap/SBA-3 from contaminated water against the cycles of $4 \mathrm{~h}$.

\section{Conclusion}

SBA-3 nanostructured materials have good structural and textural properties, useful to acts as hosts for incorporating nanocrystals of hydroxyapatite, forming active HaP/SBA3 composite. By HRTEM. FTIR, XRD, we found that the HaP nanocrystals are within the hosts, and not on the external surface, indicating good incorporation of nanocrystals in the host with sizes lower than $2 \mathrm{~nm}$. The superior efficiency of $\mathrm{HaP} / \mathrm{SBA}-3$ relative to HaP/MCM-41 and HaP/SBA-15 could also be because SBA-3 has a higher abundance of $\mathrm{Si}-\mathrm{OH}$ groups than the other reservoirs. Thus $\mathrm{Ca} 2+$ ofHaP became anchored onto the large quantity of siloxane species composed of adjacent silanol groups contained in SBA-3, enhancing the chance of more growth sites for the smaller HaP nanocrystals, because of the small pore size of SBA-3, than the other mesoporous silica supports studied (4 and $6 \mathrm{~nm}$ of MCM-41 and SBA-15 respectively).

\section{Acknowledgment}

JC, OAA, CONICET researchers, UTN-FRC. The authors thank FONCyT. PICT 2017-2021 1740.

\section{References}

1. Kresge CT, Leonowicz ME, Roth W, Vartulli JC, Beck J (1992) Ordered mesoporous molecular sieves synthesized by a liquid-crystal template mechanism. Nature 359: 710-712.
2. Beck JS, Vartuli JC, Roth WJ, Leonowicz ME, Kresge CT, et al. (1992) A new family of mesoporous molecular sieves prepared with liquid crystal templates. J Am Chem Soc 114: 10834-10843.

3. Ryoo R, Joo SH, Ju S (1999) Synthesis of highly ordered carbon molecular sieves via template-mediated structural transformation. J Phys Chem B 103: 7743-7746.

4. Rodriguez M, Anunziata OA, Beltramone AR, Martínez M (2021) Multiple-wall carbon nanotubes obtained with mesoporous material decorated with ceria-zirconia. Mater Lett 283: 128900.

5. Anunziata O, Beltramone A, Cussa J (2009) Hydroxyapatite/MCM41 and SBA-15 nano-composites: Preparation, characterization and applications. Materials 2: 1508-1519.

6. Gong WX, Qu JH, Liu RP, Lan HC (2012) Effect of aluminum fluoride complexation on fluoride removal by coagulation. Colloid Surf A 395: 88-93.

7. TorA (2007) Removal of fluoride from water using anion-exchange membrane under Donnan dialysis condition. J Hazard Mater 141: 814818.

8. He JY, Chen K, Cai XG, Li YL, Wang CM, et al. (2017) A biocompatible and novelly-defined Al-HAP adsorption membrane for highly effective removal of fluoride from drinking water. J Colloid Interface Sci 490: $97-$ 107.

9. Viswanathan N, Meenakshi S (2009) Role of metal ion incorporation in ion exchange resin on the selectivity of fluoride. J Hazard Mater 162 920-930.

10. Liao XP, Shi B (2005) Adsorption of fluoride on zirconium (IV)impregnated collagen fiber. Environ Sci Technol 39: 4628-4632.

11. Velazquez-Jimenez LH, Hurt RH, Matos J, Rangel-Mendez JR (2014) Zirconium-carbon hybrid sorbent for removal of fluoride from water: Oxalic acid mediated $\mathrm{Zr}$ (IV) assembly and adsorption mechanism. Environ Sci Technol 48: 1166-1174.

12. Bhatnagar A, Kumar E, Sillanpää M (2011) Fluoride removal from water by adsorption-A review. Chem Eng J 171: 811-840.

13. Zhao B, Zhang Y, Dou XM, Wu XM, Yang M (2012) Granulation of Fe-Al-Ce trimetal hydroxide as a fluoride adsorbent using the extrusion method. Chem Eng J 185-186: 211-218.

14. Vences-Alvarez E, Velazquez-Jimenez LH, Chazaro-Ruiz LF, Diaz-Flores PE, Rangel-Mendez JR (2015) Fluoride removal in water by a hybrid adsorbent lanthanum-carbon. J Colloid Interface Sci 455: 194-202.

15. Dehghani MH, Haghighat GA, Yetilmezsoy K, Mckay G, Heibati B, et al. (2016) Adsorptive removal of fluoride from aqueous solution using single- and multi-walled carbon nanotubes. J Mol Liq 216: 401-410.

16. Çengeloğlu Y, Kır E, Ersöz M (2002) Removal of fluoride from aqueous solution by using red mud. Sep Purif Technol 28: 81-86.

17. Cai HM, Xu LY, Chen GJ, Peng CY, Ke F, et al. (2016) Removal of fluoride from drinking water using modified ultrafine tea powder processed using a ball-mill. Appl Surf Sci 375: 74-84.

18. Kumar P, Pournara A, Kim KH, Bansal V, Rapti S, et al. (2017) Metalorganic frameworks: Challenges and opportunities for ion-exchange/ sorption applications. Prog Mater Sci 86: 25-74.

19. Elliot J (1994) Structure and chemistry of the apatite and other calcium orthophosphates. Elsevier, Amsterdam, and references therein.

20. Dalas E, Chrissanthopoulos A (2003) The overgrowth of hydroxyapatite on new functionalized polymers. J of Cryst Growth 255: 163.

21. Rivera-Muñoz E, Brostow W, Rodríguez R, Castaño V (2001) Growth of hydroxyapatite on silica gels in the presence of organic additives: kinetics and mechanism. Mat Res Innovat 42: 222-230. 
22. Laghzizil A, Elhrech N, Britel O, Bouhaouss A (2000) Removal of fluoride from moroccan phosphate and synthetic fluoroapatites. J of Fluoride Chem 101: 69-73.

23. Lett J, Sundareswari M, Ravichandran K, Bavani Latha M, Sagadevan S, et al. (2019) Tailoring the morphological features of sol-gel synthesized mesoporous hydroxyapatite using fatty acids as an organic modifier. RSC Adv 9: 6228-6240.
24. Anunziata OA, Martínez ML, Gomez Costa M (2010) Characterization and acidic properties of Al-SBA-3 mesoporous material. Mater Lett 64: 545-548.

25. Martínez M, Gómez M, Monti G, Anunziata OA (2011) Synthesis, characterization and catalytic activity of AlSBA-3 mesoporous catalyst having variable silicon-to-aluminum ratios. Micropor Mesopor Mater 144: 183-190.

For possible submissions Click below:

Submit Article 